\title{
Coordination on formal vs. de facto standards: a dynamic approach
}

\author{
Paul Belleflamme*
}

April 5, 2000

\begin{abstract}
Formal standards arise out of deliberations of standards-writing organizations, while de facto standards result from unfettered market processes. Therefore, the former are of a higher quality and legitimacy, but are slower to develop than the latter. To address this trade-off, we analyze a dynamic game where two players choose between one evolving formal standard and one mature de facto standard. The outcome of the game relies on the coordination mechanism used by the players, on the relative value they attach to successful coordination, and on the formal standard's performance at the end of the game.
\end{abstract}

JEL classification codes: C72, D71, L86.

Keywords: Standardization, negotiation, bandwagon.

*Department of Economics, Queen Mary and Westfield College, Mile End Road, London E1 4NS (phone: + 44207882 5587, fax: +44 208983 3580, e-mail: p.belleflamme@qmw.ac.uk, home page: http://www.qmw.ac.uk/ ugte186/). This research has been initiated when I was at CITA, University of Namur (Belgium). Financial support of the Belgian Federal Office for Scientific, Technical and Cultural Affairs (O.S.T.C. Interuniversity Poles of Attraction, Phase IV, program P4/31) is gratefully acknowledged. I would like to thank my thesis advisor, Louis Gevers, for his guidance. Discussion with Renaud Delhaye has been particularly fruitful. I am also grateful to Francis Bloch, Jacques Crémer, Frédéric Gaspart, Manfred Holler, Pierre Régibeau, Jacques-François Thisse, and Nick Vriend for their comments on earlier drafts. Errors are my own. 


\section{Introduction}

Over the past two decades, information and communications technologies (ICT) have evolved from stand-alone or closed systems to mass-market products. This development of ICT products and services, notably through the emergence of networks, has highlighted the need for compatibility and interoperability, i.e., the ability of products from different manufacturers to work one with another.

Standardization is one important way to achieve this much sought after compatibility. Standards (i.e., particular technical specifications to be adopted by everyone) may arise from different processes. A useful distinction can be made between formal and de facto standards: formal standards are mandated by government bodies or arise out of deliberation of voluntary standards-writing organizations; de facto standards, on the other hand, are produced through unfettered market processes, which are either "sponsored" or "unsponsored" (depending on whether there exists or not an identified originator with a proprietary interest). ${ }^{1}$

It is important to note that these two types of standardization process lead to standards with contrasting economic performance. As emphasized by the European Commission (1996), "standards have to follow the life-cycles of products and services, but since ICT product cycle times are short and becoming shorter, standards adopted following traditional procedures often prove inappropriate because of the lengthy procedures involved in their elaboration. In some areas, however, such as the development of basic telecommunications infrastructures, technical specifications need to have long-term stability and will constitute a guarantee that will allow users confidently to make the large investments that are needed." Lehr (1992) expresses the same idea in his analysis of the standardization process within standards development organizations (SDOs): "Open participation rules, supplemented with public notice requirements, enhance the likelihood that all relevant information will be included in the cost/benefit analyses of competing standard proposals. The technical groups provide an environment wherein industry participants may share information relatively inexpensively, thereby reducing collective uncertainty and lowering investment costs. Facilitating this communication is an important function for the SDOs; however, open participation also slows standardization." 2

\footnotetext{
${ }^{1}$ For more on this distinction, see, e.g., David and Greenstein (1990).

${ }^{2}$ David and Shurmer (1996) report that "the average time taken [by the formal standardization process] to produce a standard varies from $2 \frac{1}{2}$ years at the national level, through to $4-5$
} 
To sum up, the following trade-off confronts the ICT sector in its need for compatibility. On the one hand, formal standards should be preferred over de facto standards because they are developed through agreed, open and transparent procedures, based on a consensus of all interested parties; they present thereby a particular legitimacy and avoid the costs, associated with de facto standards, of adopting privately profitable, but socially undesirable, technologies. On the other hand, formal standards suffer a major drawback: the pace of reaching them is often too slow in a context of rapid technological progress compared to the rather quick emergence of de facto standards. As Lehr (1992) judiciously puts it, "the irony of industry standardization is that the rapid pace of technical change simultaneously increases the social costs both from delay and from adopting the wrong standards." 3

While a large literature has analyzed the market process generating de facto standards, very little attention has surprisingly been devoted to two equally important issues:on the one hand, the work and performance of formal standardization institutions and on the other hand, the potential rivalry between formal and market-driven standardization. ${ }^{4}$

Farrell and Saloner (1988) have been the first to explicitly address the former issue. They start from the finding that both processes are imperfect and they try to answer the following questions: Which is better? What does that depend on? What are the advantages and disadvantages of each? To this end, they study situations where all participants would prefer any proposed standardized outcome to the result of each going his own way, but in which they disagree on which of the standardized outcome is better. In such a framework, they discuss three common mechanisms for achieving coordination. The first involves explicit com-

\footnotetext{
years regionally, and 7 years or more at the international level."

${ }^{3}$ See also David and Shurmer (1996) for a discussion on this trade-off.

${ }^{4}$ The abundant literature on de facto standardization has stressed how positive network externalities (which refer to the idea that the performance of a standard as well as its utility increases with the growth of the community of users) raise technical problems (equilibrium may not exist, or multiple equilibria may exist) and affect market performance (the fundamental theorems of welfare economics may not apply; in particular, markets may generate too little or too much standardization); recent surveys of this literature are found in Katz and Shapiro (1994), Besen and Farrell (1994), Economides (1996) and Matutes and Régibeau (1996).The literature about formal standardization is, comparatively, rather scarce and often of a more descriptive or political nature (see Greenstein, 1992; Lehr, 1992; Weiss and Cargill, 1992; Foray, 1994; David and Shurmer, 1996). Exceptions are Farrell and Saloner (1988), Swann and Shurmer (1994), Goerke and Holler (1995).
} 
munication and negotiation before irrevocable choices are made: it represents the formal standardization process. The second mechanism, by contrast, involves no explicit communication and depends on unilateral irrevocable choices: it succeeds if one agent chooses first and the others follows, which corresponds to de facto standardization. The model they build allow them to reach the following results: formal standardization is more likely to achieve fruitful coordination; moreover, although this mechanism is slower, it outperforms the market mechanism, even when the value of speed is taken into account. They also examine a hybrid of the first two mechanisms which is shown to perform even better than formal standardization.

Swann and Shurmer (1994) tackle the latter issue. They underline that formal and de facto standards can in a sense be seen as rival standards: "the institutional standard may be better but there is a longer wait for it, while current proprietary standards-imperfect as they may be-are available sooner." To account for the rivalry between the two types of standards, they use a simulation model of the emergence of standards in the spreadsheet software market and examine the effects of some simple policy interventions by a standards-setting institution. They examine the case where an institution is attempting to 'sell' its standard by pre-announcement, while individual producers market their own proprietary designs before the institutional standard is available. They show that an improved quality of institutional standard will usually increase average consumer surplus and the institution's market share, but earlier pre-announcements need not necessarily achieve this.

With respect to these two articles, the present paper stands somewhere inbetween: it considers the question addressed by Swann and Shurmer (i.e., the rivalry between de facto and formal standard, rather than the comparison of the relative speed, efficacy and payoffs of the two standardization processes), but follows Farrell and Saloner's framework (i.e., a repeated coordination game, rather than a non-strategic simulation model). Specifically, this paper considers how users choose, in a dynamic setting, between competing standards that result from different standardization processes. The interest for this issue is aroused by two empirical findings:

- formal standards often appear under the form of successive versions that are increasingly effective;

- these successive versions have to compete with well-established de facto 
standards.

A first concrete example that fits this description is the development of the Small Computer System Interface (SCSI) standard. SCSI is a parallel interface standard used by Apple Macintosh computers, PCs, and many UNIX systems for attaching peripheral devices (e.g., printers, disk drives, display monitors, keyboards, mice, ...) to computers. Although SCSI is a formal standard established by the American National Standards Institute (ANSI), there are several variations of it: the original SCSI standard was approved by ANSI in 1986 and was followed by SCSI-2 in 1994; the SCSI-3 specification was drafted in 1996 and should be approved soon. So, SCSI has grown and evolved to keep pace with the demands of the most sophisticated systems, to recognize virtually every peripheral type and to take advantage of newer hardware and more intelligent controllers. These successive variations of SCSI have been, or are currently, competing with other, de facto, interface standards. According to Webopedia", "While SCSI has been the standard interface for Macintoshes, the iMac comes with IDE, a less expensive interface, in which the controller is integrated into the disk or CD-ROM drive. Other interfaces supported by PCs include enhanced IDE and ESDI for mass storage devices, and Centronics for printers. You can, however, attach SCSI devices to a PC by inserting a SCSI board in one of the expansion slots. Many high-end new PCs come with SCSI built in. Note, however, that the lack of a single SCSI standard means that some devices may not work with some SCSI boards." As illustrated by the latter two sentences, the coexisting standards are imperfectly compatible with each other; moreover, even successive SCSI interfaces are not fully backward compatible. ${ }^{6}$

The diffusion of Electronic Data Interchange (EDI) provides a second example. EDI is a technology that allows business documents and other communications to be automatically interpreted by computers in different organizations, thereby avoiding the time-consuming and error-inducing rekeying of information. Standardized business messages are clearly the precondition for such an automated exchange and interpretation of message contents. This task is performed by 'message standards', that is, standardized ways of describing the component parts of

\footnotetext{
${ }^{5}$ See http://webopedia.internet.com/Hardware/Buses/SCSI.html

${ }^{6}$ For an authorized opinion on that matter, see "Confusion over SCSI standards", PC Magazine Online (5/5/98), at http://www.zdnet.com/pcmag/pctech/content/solutions/ hw1709a.htm.
} 
trading documents and of grouping and presenting them in the form of messages or trade information (invoice, purchase order and so forth). Various message standards coexist nowadays: on the one hand, a number of de facto standards have been elaborated by large companies or self-sufficient trading communities to address their specific business needs; on the other hand, public institutions have also taken an active part in the definition of formal standards which go beyond the sectorial needs. In the latter category, the most prominent formal standard is EDIFACT (for 'Electronic Data Interchange for Administration, Commerce and Transport'): this standard is sponsored, since the late 1980's, by the International Standards Organization (ISO) and the United Nations Economic Commission for Europe (UN/ECE) and aims to become the single international EDI standard, flexible enough to meet the needs of governments and private industry. To achieve this goal, however, is anything but simple and the elaboration of EDIFACT is inevitably a slow process. So, since the approval of the first message in 1988, the standard has constantly been widened to cover a growing number of industry sectors. To date around 160 messages have been approved as "United Nations Standard Messages" (UNSMs) and approximately 100 have been approved as "Messages in development"; standard directory sets containing UNSMs are issued twice each year. ${ }^{7}$

A third example can be found in the standardization process of the $56 \mathrm{Kbps}$ modems. The standard for 56Kbps modems, known as V.90, was completed by the International Telecommunication Union in February 1998 and officially stamped for approval in September 1998. Industry analysts concurred in pointing out that this standard was coming too late. The lengthy standard battle which preceded the approval was deemed to have long-lasting damaging effects. Before V.90 was established, consumers had to choose between 56 Kbps modems using either 3Com's x2 or Rockwell's K56 flex technology. Users then had to find an Internet Service Provider (ISP) that supported the modem technology they had purchased. It also caused many ISPs to refrain from supporting 56 Kbps modems because of the reigning confusion at that time. Still, the formal adoption of the V.90 standard did not appear as a panacea for modem vendors. While hailed by industry players as a solution to compatibility issues between the two existing technologies, V.90 was still exhibiting teething issues, as glitches

\footnotetext{
${ }^{7}$ Belleflamme (1999) applies the methodology developed in this paper to the specific case of EDI.
} 
with the iMac from Apple computer showed. Additionally, there were reports of other lingering compatibility issues between 3-Com and Rockwell-based V.90 technologies. ${ }^{8}$ Boardwatch Magazine described the situation that prevailed in March 1998 in the following way: "We think 56 Kbps modems will be getting better. And now we have a standard-V.90. But the battle is not precisely over. There will likely be ongoing performance differences in this round (...). [V.90 modems] may interoperate, but you will most likely get the best performance using a modem and technology that matches the one supported by your ISP." 9

The present paper develops a specific model to analyze the dynamic choice Between de facto and formal standards in situations where the above two findings are observed. Specifically, I assume that two players have to choose between one formal and one de facto standard; both players prefer to adopt a common standard but their preferences diverge about which particular standard to choose; in game-theoretic jargon, they play a game having the features of the classic 'battle of the sexes' (which is subsequently denoted by BoS). ${ }^{10}$ Moreover, I assume that the de facto standard is 'mature', in the sense that it has constant performance over the game period, while the formal standard undergoes a development process: successive versions of increasing value are released and, in particular, the formal standard performs worse today than the de facto standard, but is known (with certainty) to perform better in the future. Since players have a strong incentive to reach an agreement, it is assumed that they will follow some dynamic coordination mechanism. In particular, in the spirit of Farrell and Saloner (1988), I consider either that players can explicitly communicate and negotiate before irrevocable choices are made, or that no such communication is allowed and that coordination depends instead on unilateral irrevocable choices. These two mechanisms are compared with a benchmark that selects the solution with the highest sum of players' payoffs.

It turns out from the resolution of this dynamic game that the outcome crucially relies on three key factors: (i) the particular coordination mechanism used

\footnotetext{
${ }^{8}$ See "V.90 modem standard approved", by S. Miles at http://www.news.com/News/ Item/0,4,26486,00.html (17/09/1998).

${ }^{9}$ See "The $56 \mathrm{~K}$ modem battle", by J. Richard at http://www.boardwatch.com/mag/ 98/mar/bwm24.html.

${ }^{10}$ In this classic coordination game, a man and a woman are choosing between the ballet and a football game. The main concern of each is to be in the company of the other (regardless of where they go); but he prefers the ballet and she the football game (or vice versa).
} 
by the players, (ii) the relative value for the players of successful coordination, and (iii) the performance of the formal standard at the end of the game. Regarding the adoption of the formal standard, the higher the latter two values, the lower the minimal degree of patience players should have to both adopt this standard. In terms of comparison with the benchmark, both coordination mechanisms are imperfect but entail contrasting failures: verbal negotiation leads to an excessive adoption of the formal standard, while unilateral adoption excessively favors the de facto standard.

At the start, I wish to stress that the purpose of this paper is more to highlight the interplay between the above three factors and to draw out some policy implications, and so the model presented is voluntarily kept as simple as possible. The rest of the article is organized as follows. Section 2 outlines the model; Sections 3 and 4 respectively analyze the 'negotiation' and 'bandwagon' games; Section 5 compares the results of the two games with the benchmark and derives some policy implications; Section 6 concludes.

\section{The model}

\subsection{Basic setup}

In the spirit of Farrell and Saloner (1988), I study a two-player game which has the payoff structure of the classic BoS: the players (noted 1 and 2) have to choose one of two possible standards (noted $X$ and $Y$ ). The situation is such that each player would prefer any proposed coordinated outcome to the result of each going her own way; however, the players disagree on which of the coordinated outcomes is better (say that player 1 [resp. 2] prefers coordination on standard $X$ [resp. $Y]$ ). The interesting issue is whether the players will manage to coordinate their choices and, if they do, on which standard.

To address this issue, I assume that there are three periods in the game and that the choice of standards by the players can be achieved through two different dynamic procedures. In both procedures, periods 1 and 2 are 'coordination rounds' and period 3 is the deadline. In other words, while the period 3 stage game is common, the form of the game in periods 1 and 2 depends on the coordination mechanism used by the players. Two such mechanisms are considered and compared.

A first coordinating principle is to use verbal negotiation. In the Negotiation 
game, the players have two chances to reach verbal agreement before the game of period 3 must be played. That is, each player can (simultaneously) announce 'insist' (i.e., stand firm with one's preferred standard) or 'concede' (i.e., accept to adopt the other player's preferred standard); if just one player insists, then she gets her way, while if both insist or if neither does, then they meet again in the following period. If no agreement has been reached after two periods, then the players play the game of period 3 .

A second possible coordinating principle is to use unilateral adoption rather than negotiation; we have then a market mechanism which is usually called 'bandwagon' in the literature (see, for instance, Farrell and Saloner, 1985) In this so-called Bandwagon game, each player can either commit unilaterally to her preferred standard (and hope that the other will then follow) or simply wait. In contrast with the previous mechanism, it is only when both players decide to wait that they meet again at the following period.

The remaining issue is to determine which coordination mechanism is better. I use, as a benchmark, the solution that maximizes the sum of the players' (discounted) payoffs (see Section 5).

\subsection{Payoff structure}

As in Farrell and Saloner (1988), let $a$ denote the value for a player of being on one's preferred standard, and $c$ the additional value of successful coordination. Because each player would prefer any proposed coordinated outcome to the result of each going her own way, it is assumed that $c>a>0$ (since otherwise each player simply adopts her preferred alternative).

Now, unlike in Farrell and Saloner (1988), suppose that in period $t$ the values $a$ and $c$ are multiplied by a factor $x_{t}$ if the player adopts standard $X$, or by a factor $y_{t}$ if the player adopts standard $Y$. These additional factors allow the two standards to offer benefits that evolve differently through time; in particular, it is assumed that standard $X$, a de facto standard, is 'mature' (in the sense that its performance is constant over the game period) while standard $Y$, a formal standard, undergoes a development process (and, in particular, performs worse than $X$ today but is known to perform better in the future). Accordingly, the following assumptions are made:

- $x_{1}=x_{2}=x_{3}=x$ (i.e., standard $X$ 's performance is constant); 
- $y_{1}<x ; y_{2}=x ; y_{3}>x$ (i.e., the first version of standard $Y$ lags behind standard $X$, the second version has just caught up and the third version is ahead of $X)$;

- without any loss of generality, $x=1$.

It is important to note that, as in Farrell and Saloner (1988), $a$ and/or $c$ accrue to a player once and for all, at the time she actually adopts a particular standard. It is also assumed that payoffs are discounted for each period that decisions are postponed. ${ }^{11}$ The passage of time has thus two opposite effects in the model: on the one hand, because of discounting, players would rather reach an agreement sooner than later; on the other hand, technical progress and the formal standardization process make standard $Y$ 's adoption more attractive tomorrow than it is today.

One can easily draw a parallel between the previous set of assumptions and the two concrete examples given in Section 1.

- As far as EDI is concerned, a concrete situation that could square with the assumptions is the following. Players are firms: firm 1 is a car manufacturer and firm 2, an upholsterer. Because firm 1 is going to equip its new car with seats manufactured by firm 2, the two firms wish to agree on a common EDI standard; nevertheless, each firm would prefer that the standard elected be the one used in its own industry: the mature ODETTE standard in the automotive industry (i.e., standard $X$ ), or the evolving EDIFACT standard in the textile industry (i.e., standard $Y$ ). The following interpretation can then be given to explain the formation of the payoffs. It is emphasized in the literature that the economic advantages that can be derived from the use of EDI are positively related to the volume of the message exchange. We can then say that (i) $c$ and $a$ are, respectively, the total number of transactions firms 1 and 2 are going to conduct with each other, or with other trading partners, during their business relationship, and that (ii) $x_{t}$ and $y_{t}$ are respectively the per transaction benefit derived from the use of standard $X$ or $Y$ if the standard is adopted in period $t$. It must be stressed that the timing of adoption determines not only when the payoffs accrue (firms derive benefits from EDI once they have adopted a standard), but

\footnotetext{
${ }^{11}$ I assume (for simplicity) that the two players have the same discounting factor, $\delta \in[0,1]$ (the closer $\delta$ from 1 , the more patient the players).
} 
also how much firms earn (it is indeed assumed that the firms that adopt standard $Y$ at time $t$ get locked into the specific generation of $Y$ available at that time).

- Regarding parallel interface standards, the two players might be firms that respectively produce computers and peripheral devices. They have a strong incentive to agree on a common interface standard to ensure the interoperability of their products. They have to choose between the de facto standard $X$ (say, ESDI $)^{12}$ and the formal standard $Y$ (SCSI), for which they have divergent preferences (because of existing relationships with other commercial partners). This choice is complicated by the increasing performance of SCSI over time and by the relative lack of backward compatibility between its successive versions. A similar story can be told about the choice between competing 56 Kbps modem technologies made by Internet Service Providers and by modem makers.

I am now in position to delineate the whole game tree; this is done in Figure 1 for the negotiation game, and in Figure 2 for the bandwagon game.

\section{(Insert Figures 1 and 2 about here)}

For the sake of illustrating the payoff structure, let us detail the stage game of period 3 which is common to the two settings. This game is called the 'final game' since it will be played if the players can reach no agreement in period 1 or 2 ; it is represented by the payoff matrix of Table $1 .{ }^{13}$

Table 1: The final game

\begin{tabular}{l|c|c|}
\multicolumn{1}{c}{$\mathbf{X}$} & \multicolumn{1}{c}{$\mathbf{Y}$} \\
\cline { 2 - 3 } $\mathbf{X}$ & $a+c, c$ & $a, y_{3} a$ \\
\cline { 2 - 3 } $\mathbf{Y}$ & 0,0 & $y_{3} c, y_{3}(a+c)$ \\
\cline { 2 - 3 } & &
\end{tabular}

\footnotetext{
${ }^{12}$ ESDI stands for Enhanced Small Device Interface, an interface standard developed by a consortium of the leading personal computer manufacturers for connecting disk drives to PCs.

${ }^{13}$ In this matrix, as well as in all of the following matrices, player 1's choices appear in rows, and player 2's in columns; the first entry in each box is player 1's payoff, and the second is player 2's.
} 


\subsection{Equilibrium concept and resolution of the final game}

I now turn to the resolution of the dynamic game. The equilibrium concept I use is subgame-perfection. One important comment is in order about the method I follow to derive a subgame-perfect equilibrium. Because the stage games (in periods 1 to 3 ) have the features of the BoS, multiple pure-strategy Nash equilibria (PSNE) are often encountered. To go on with backward induction, I need thus to apply some criterion to select a unique Nash equilibrium to be considered as the solution of the stage game.

My approach contrasts with the one followed by Farrell and Saloner (1988) who are reluctant to use any of the PSNE; they argue that "these pure-strategy Nash equilibria are quite unrealistic without a convincing story of the mechanics of coordination (...) To model institutions that promote coordination, therefore, we must use a solution that allows for the possibility of failure of coordination. Failure might be expected if there is no preplay negotiation and if the players are symmetric so that (at least ex ante) they can be expected to behave in the same way. An obvious way to model such symmetric behavior is by focusing on the symmetric equilibrium, which involves mixed strategies: each player sometimes insists on his or her own way, and sometimes yields" (p. 238). Here, because of payoff asymmetry, this argument in favor of the mixed-strategy equilibrium seems undermined: players are no longer symmetric and cannot thus be expected to behave in the same way; for the same reason, one of the two PSNE may now appear as focal. It seems thus legitimate to use a criterion to select among the multiple equilibria.

The criterion I apply is risk dominance; this criterion has been axiomatically developed by Harsanyi and Selten (1988) for the case of pure-strategy equilibria in 2-by-2 games. Roughly speaking, the idea behind this concept is that the players are more likely to focus on the equilibrium which consists of the less risky choice for them. ${ }^{14}$ The rationale for using this criterion is threefold.

1. There is the relevance of the criterion in the case of coordination games

\footnotetext{
${ }^{14}$ Harsanyi (1995) explains the notion of risk that is at stake here: "A rational player will try to choose a strategy that is a best reply to the other players' strategies. But he will incur the strategic risk that the strategy he actually chooses will not have this property because his expectations about the other players' strategies will turn out to have been mistaken." More formally, the less risky equilibrium is the one with the widest 'basin of attraction', which has to be understood as the range of randomized strategies for which the pure strategies composing the PSNE are optimal (see the Appendix).
} 
like the BoS, as revealed by experimental evidence: Cabrales et al. (2000) have recently given support for the use of risk dominance as an equilibrium selection in the BoS; they further show that the higher the degree of asymmetry of the game, the higher the predictive power of the risk dominance criterion. ${ }^{15}$

2. There is the bite of the criterion. Most standard criteria of selection among multiple equilibria (such as perfectness, properness, strategic stability and Pareto dominance) may fail having any selective power in the stage games considered here. The criterion of risk dominance is an exception: when there are two PSNE in a stage game, the payoff asymmetry (induced by the evolution through time of standard $Y$ 's performance) always allows us to state that one PSNE 'risk-dominates' the other.

3. There is the convenience of the criterion. As will become clear below, the application of risk dominance as a selection criterion yields a clear-cut and intuitive description of the equilibrium path in all possible configurations of parameters. Keeping in mind that the objective of the paper is to highlight particular aspects of the rivalry between formal and de facto standards, the clarity of the results plays a decisive role in the choice of the model specification and resolution. ${ }^{16}$

In what follows, as a reminder that risk-dominance is applied to select among multiple PSNE in the stage games, the equilibrium will be called Risk-Dominant Subgame Perfect (RDSP). Solving the game backward, I start thus with the stage game of period 3 which is common to the two coordination mechanisms; it is represented by the payoff matrix of Table 1. From the assumptions that (i) standard $Y$ performs better than standard $X$ at the deadline (i.e., $y_{3}>1$ ), and (ii) the value of coordination is higher than the value of adopting one's preferred standard (i.e., $c>a$ ), it follows that $(Y, Y)$ is a PSNE of the final game. The other possible PSNE is $(X, X)$, provided that $y_{3}<c / a$. There are thus two cases to distinguish: (i) if $y_{3}>c / a$, then $(Y, Y)$ is the unique equilibrium in the final game; (ii) otherwise, $(X, X)$ and $(Y, Y)$ are both PSNE.

\footnotetext{
${ }^{15}$ See also, e.g., Cooper et al. $(1989,1992,1993)$ for other experimental research on similar games .

${ }^{16}$ In contrast, the selection of the mixed-strategy Nash equilibrium in place of one of the two PSNE leads to a division of the parameter space and to equilibrium paths that are hard to interpret intuitively.
} 
As announced above, I apply the risk-dominance criterion to select one of the two PSNE in the latter case. Straightforward computations (based on the methodology described in the Appendix) show that $(Y, Y)$ is the risk-dominant equilibrium. It seems thus legitimate to consider that if the deadline is reached, the players will coordinate their choices on the formal standard $Y$ (because this situation will be either the unique PSNE in the final game, or the focal PSNE in terms of risk dominance).

We can now turn to the resolution of the subgames corresponding to the two coordination mechanisms.

\section{Negotiation game}

In the negotiation game, the coordinating principle is to use verbal negotiation: the players have two chances to reach verbal agreement before the final game must be played. That is, each player can (simultaneously) announce 'insist' (i.e., stand firm with one's preferred standard) or 'concede' (i.e., accept to adopt the other player's preferred standard); if just one player insists, then he gets his way, while if both insist or if neither does, then they meet again in the following period. If no agreement has been reached after two periods, then the players play the final game (see Figure 1). Folding back the game tree, we first solve the stage game of period 2 and then the stage game of period 1.

\subsection{Period 2}

The payoffs for the negotiation game in period 2 are reported in the matrix of Table 2 (where the assumption that $y_{2}=1$ has been taken into account). Because the players meet again in period 3 when they both insist or concede, the payoffs in these two cells of the matrix correspond to the discounted period 3 equilibrium payoffs: $\left(\delta y_{3} c, \delta y_{3}(a+c)\right) \cdot{ }^{17}$

Comparing the payoffs in Table 2, we can determine which PSNE obtain according to the value of $\delta$; in the regions where multiple PSNE are observed, the risk-dominance criterion is applied. The following lemma summarizes the

\footnotetext{
${ }^{17}$ Recall that no payoff accrues to the players until they have adopted one or the other standard; therefore, if they both concede or insist in period 2 (meaning that they have also done so in period 1), they only earn payoffs in period 3 .
} 
Table 2: Negotiation game in period 2

\begin{tabular}{c|c|c|}
\multicolumn{1}{c}{ Concede } & \multicolumn{1}{c}{ Insist } \\
\cline { 2 - 3 } Insist & $a+c, c$ & $\delta y_{3} c, \delta y_{3}(a+c)$ \\
\cline { 2 - 3 } Concede & $\delta y_{3} c, \delta y_{3}(a+c)$ & $c, a+c$ \\
\cline { 2 - 3 } & &
\end{tabular}

results. ${ }^{18}$

Lemma 1 The (unique or risk-dominant) PSNE in the period 2 negotiation game is

- (Concede, Insist) for $0 \leq \delta \leq y_{3}^{-1}$;

- (Insist, Insist) for $y_{3}^{-1} \leq \delta \leq 1$.

In words, in the case where period 2 is reached, the players will either coordinate on standard $Y$ if they are relatively impatient or prefer to meet once again if they are relatively patient. Here, players are said to be patient when their discounting factor is such that $\delta y_{3}>y_{2}=1$; that is, when standard $Y$ 's performance are higher (in present value) in period 3 than in period 2 .

\subsection{Period 1}

Let $V_{i}(2)$ denote the value for player $i$ of having two meetings remaining; this 'continuation payoff' is what player $i$ gets in period 1 if both players concede or insist (that is, the discounted period 2 equilibrium payoff). From Lemma 1, we have the following values:

- if $0 \leq \delta \leq y_{3}^{-1}$, then $V_{1}(2)=\delta c$ and $V_{2}(2)=\delta(a+c)$;

- if $y_{3}^{-1} \leq \delta \leq 1$, then $V_{1}(2)=\delta^{2} y_{3} c$ and $V_{2}(2)=\delta^{2} y_{3}(a+c)$.

The matrix of Table 3 describes the payoffs for the negotiation game in period 1.

I will not get down here to the nitty-gritty of solving this stage game. I confine myself in stating what (unique or risk-dominant) PSNE obtains according to the

\footnotetext{
${ }^{18}$ The proof of this lemma, as well as those of other lemmas and propositions, can be found in an appendix available from the author.
} 
Table 3: Negotiation game in period 1

\begin{tabular}{c|c|c|}
\multicolumn{1}{c}{} & \multicolumn{1}{c}{ Concede } & \multicolumn{1}{c}{ Insist } \\
\cline { 2 - 3 } Insist & $a+c, c$ & $V_{1}(2), V_{2}(2)$ \\
\cline { 2 - 3 } Concede & $V_{1}(2), V_{2}(2)$ & $y_{1} c, y_{1}(a+c)$ \\
\cline { 2 - 3 } & &
\end{tabular}

values of the parameters. To organize the results, I distinguish between four main cases, defined in terms of standard $Y$ 's performance at the beginning and at the end of the game. As far as starting performance is concerned, the value of $y_{1}$ has to be compared with the ratio $c /(a+c)$ which gives the relative value for a player of successful coordination: for $y_{1}$ just equal to $c /(a+c)$, player 2 gets the same payoff in period 1 whether choices are coordinated on $X$ or on $Y$-i.e., $c$ vs. $y_{1}(a+c)$. Similarly, final performance $y_{3}$ is appraised with respect to the inverse ratio, $(a+c) / c$ : for $y_{3}$ just equal to $(a+c) / c$, player 1 gets the same payoff in period 3 whether choices are coordinated on $X$ or on $Y$-i.e., $a+c$ vs. $y_{3} c$. Accordingly, I will refer to the following taxonomy:

Case LL (Low/Low) : $y_{1}<c /(a+c)$ and $y_{3}<(a+c) / c$;

Case LH (Low/High) : $y_{1}<c /(a+c)$ and $y_{3}>(a+c) / c$;

Case HL (High/Low) : $y_{1}>c /(a+c)$ and $y_{3}<(a+c) / c$;

Case HH (High/High) : $y_{1}>c /(a+c)$ and $y_{3}>(a+c) / c$.

In what follows, I will focus on cases LL, LH and HH; case HL is omitted because it is not really relevant for our purpose since it assumes away the difference in standards' performance we precisely want to address.

The main results gathered from the resolution of the period 1 negotiation game can be summarized as follows. When the initial performance of standard $Y$ is low (cases LL and LH), the players either coordinate on standard $X$ (for 'low' values of the discounting factor, player 1 insists while player 2 concedes), or go for a second meeting (for 'high' values of $\delta$, they both insist). In contrast, when $Y$ 's initial performance is high (case $\mathrm{HH}$ ), the latter two outcomes are observed only for 'extreme' values of $\delta$, while for 'intermediate' values of $\delta$, the players prefer to coordinate on standard $Y$ (since at the equilibrium, player 1 concedes and player 2 insists). 
The boundaries between low, intermediate and high values of the discounting factor vary from one case to the other but always have some instructive intuitive meaning. In case LL, the cutoff is where $\delta=c /(a+c)$; remembering that standard $Y$ 's performance in period $2\left(y_{2}\right)$ is, by assumption, equal to one, the latter equality is equivalent to $\delta y_{2}(a+c)=c$, which means that, in present value, player 2 is indifferent between coordinating on $Y$ in period 2 or on $X$ in period 1 . Similarly, in case LH, the equality between $\delta$ and the cutoff can be rewritten as: $\delta^{2} y_{3}(a+c)=c$, which is the indifference condition for player 2 between coordinating on $Y$ in period 3 or on $X$ in period 1 .

In case $\mathrm{HH}$, the cutoff between 'intermediate' and 'high' values of $\delta$ also expresses a condition about the evolution of $Y$ 's performance: $\delta=\sqrt{y_{1} / y_{3}} \Longleftrightarrow$ $\delta^{2} y_{3}=y_{1}$ is equivalent to say that $Y$ 's performance (in discounted value) is the same in period than in period 1. ${ }^{19}$ Finally, the cutoff between 'low' and 'intermediate' values of $\delta$ follows from a technical condition for the risk-dominance relation between multiple PSNE. Formally, define the following threshold:

$$
\gamma=\frac{c(a+c)\left(1-y_{1}^{2}\right)}{a^{2}+2 c(a+c)\left(1-y_{1}\right)} .
$$

It can be shown that this threshold plays a critical role to determine whether (Insist, Concede) or (Concede, Insist) is risk-dominant in the regions of parameters where they are both PSNE. More precisely, in the case where $y_{3}<\gamma^{-1}$, the relevant cutoff is $\gamma$; otherwise, the relevant cutoff is $\sqrt{\gamma / y_{3}}$.

In summary, let us adopt the following notation for the various cutoffs (where the subscript $n$ stands for 'negotiation'):

$$
\begin{aligned}
& \bar{\delta}_{n}= \begin{cases}c /(a+c) & (\text { case LL }), \\
\sqrt{c / y_{3}(a+c)} & (\text { case LH }) ;\end{cases} \\
& \tilde{\delta}_{n}=\sqrt{y_{1} / y_{3}}(\text { case HH }) ; \\
& \hat{\delta}_{n}=\left\{\begin{array}{ll}
\gamma & \text { if } y_{3}<\gamma^{-1}, \\
\sqrt{\gamma / y_{3}} & \text { otherwise; }
\end{array}\right. \text { (case HH). }
\end{aligned}
$$

Lemma 2 states the results for the negotiation game in period 1.

Lemma 2 The (unique or risk-dominant) PSNE in the period 1 negotiation game is

\footnotetext{
${ }^{19}$ Rewriting the discounting factor as $\delta=1 /(1+r)$, where $r$ is the (per period) interest rate at which payoffs can be invested, the two expressions are equivalently interpreted as equalities between the interest rate and the (per period) rate of growth of $Y$ 's performance.
} 
- (Insist, Concede) for $0 \leq \delta \leq \bar{\delta}_{n}$ in cases $L L$ and $L H$, and for $0 \leq \delta \leq \hat{\delta}_{n}$ in case $\mathrm{HH}$;

- (Concede, Insist) for $\hat{\delta}_{n} \leq \delta \leq \tilde{\delta}_{n}$ in case $H H$;

- (Insist, Insist) for $\bar{\delta}_{n} \leq \delta \leq 1$ in cases LL and LH, and for $\tilde{\delta}_{n} \leq \delta \leq 1$ in case $\mathrm{HH}$.

\subsection{Summary}

We can now collect the previous results in order to answer the following question: on which standard - and when - do the players coordinate in the negotiation game? The following proposition states the answer for all combinations of parameter values.

Proposition 3 The standard adopted by the players and the timing of adoption at the RDSP equilibrium of the negotiation game depend on the values of the parameters in the following way.

- For low discount factors, players adopt standard $X$ in period 1 (i.e., for $0 \leq \delta \leq \bar{\delta}_{n}$ in cases $L L$ and $L H$, and for $0 \leq \delta \leq \hat{\delta}_{n}$ in case $\left.H H\right)$.

- For intermediate discount factors, players adopt standard $Y$ either in period 1 (i.e., for $\hat{\delta}_{n} \leq \delta \leq \tilde{\delta}_{n}$ in case HH) or in period 2 (i.e., for $\bar{\delta}_{n} \leq \delta \leq y_{3}^{-1}$ in case $L L)$.

- For high discount factors, players adopt standard $Y$ in period 3 (i.e., for $y_{3}^{-1} \leq \delta \leq 1$ in case $L L$, for $\bar{\delta}_{n} \leq \delta \leq 1$ in case $L H$, and for $\tilde{\delta}_{n} \leq \delta \leq 1$ in case $H H)$.

In other words, Proposition 3 shows that very impatient players immediately coordinate on $X$ and very patient players wait for the deadline and coordinate on $Y$, whatever the (initial and final) performance of standard $Y$. In contrast, ambiguous outcomes prevail when players are relatively (im)patient: according to $Y$ 's performance, the players either coordinate immediately (on $X$ or on $Y$ ) or adopt $Y$ in period 2. Let us now analyze whether similar results obtain under the alternative coordinating principle. 


\section{Bandwagon game}

Unilateral adoption is now substituted for negotiation as the coordinating principle used by the players. Under this market mechanism (called 'bandwagon'), each player can either commit unilaterally to its preferred standard (and hope that the other will then follow) or simply wait. Hence, in contrast with the negotiation game, it is only when both players decide to wait (i.e., 'concede', in the terminology of the negotiation game) that they meet again at the following period. As above, the game is solved by backward induction, starting with period 2 .

\subsection{Period 2}

The payoffs for the bandwagon game in period 2 are reported in the matrix of Table 4 . The difference with the negotiation game (see Table 2) is that there is now only one cell where the discounted period 3 equilibrium payoffs are reported.

Table 4: Bandwagon game in period 2

\begin{tabular}{c|c|c|}
\multicolumn{1}{c}{} & \multicolumn{1}{c}{ Wait } & Commit to $\mathbf{Y}$ \\
\cline { 2 - 3 } Commit to $\mathbf{X}$ & $a+c, c$ & $a, a$ \\
\cline { 2 - 3 } Wait & $\delta y_{3} c, \delta y_{3}(a+c)$ & $c, a+c$ \\
\cline { 2 - 3 } & &
\end{tabular}

It is straightforward that both players committing to their preferred standard cannot be a PSNE of this stage game (because, by assumption, $c>a$ ). Furthermore, it can be shown that if (Wait, Commit to $Y$ ) is a PSNE, it is necessarily risk-dominated by (Commit to $X$, Wait). There are thus two possible outcomes for the game: either (Commit to $X$, Wait) if $\delta<\bar{\delta}_{b} \equiv(a+c) /\left(y_{3} c\right)$, or (Wait, Wait) otherwise. It is clear, however, that the latter PSNE cannot be observed if $(a+c) /\left(y_{3} c\right)>1 \Longleftrightarrow y_{3}<(a+c) / c$ (i.e., in case LL). The following lemma summarizes these results.

Lemma 4 The (unique or risk-dominant) PSNE in the period 2 bandwagon game is

- (Commit to $X$, Wait) in case $L L$, for all $\delta \in[0,1]$, and in cases $L H$ and $H H$, for $0 \leq \delta \leq \bar{\delta}_{b}$; 
- (Wait, Wait) in cases LH and HH, for $\bar{\delta}_{b} \leq \delta \leq 1$.

A major difference with respect to the negotiation mechanism immediately appears: whatever their degree of patience, the players will not wait for the deadline if the final performance of standard $Y$ is poor (more precisely, if $y_{3}$ is such that player 1 is better off in period 3 when choices are coordinated on $X$ rather than on $Y$-i.e., if $a+c>y_{3} c$ ). The deadline will be reached only if (i) $Y$ 's final performance is high, and (ii) players are relatively patient.

\subsection{Period 1}

Let $W_{i}(2)$ denote the value for player $i$ of having two periods remaining; this 'continuation payoff' is the discounted period 2 equilibrium payoff that player $i$ gets in period 1 if both players decide to wait. From Lemma $4,\left(W_{1}(2), W_{2}(2)\right)$ can be equal either to $(\delta(a+c), \delta c)$ or to $\left(\delta^{2} y_{3} c, \delta^{2} y_{3}(a+c)\right)$. The matrix of Table 5 describes the payoffs for the period 1 bandwagon game.

Table 5: Bandwagon game in period 1

\begin{tabular}{c|c|c|}
\multicolumn{1}{c}{} & \multicolumn{1}{c}{ Wait } & \multicolumn{1}{c}{ Commit to Y } \\
\cline { 2 - 3 } Commit to & $a+c, c$ & $a, y_{1} a$ \\
\cline { 2 - 3 } & $W_{1}(2), W_{2}(2)$ & $y_{1} c, y_{1}(a+c)$ \\
\cline { 2 - 3 } & &
\end{tabular}

Defining $\hat{\delta}_{b}$ as $\sqrt{(a+c) /\left(y_{3} c\right)}$, I can state the main results drawn from the resolution of this game as follows. ${ }^{20}$

Lemma 5 The (unique or risk-dominant) PSNE in the period 1 bandwagon game is

\footnotetext{
${ }^{20}$ Note that the taxonomy developed for the negotiation game cannot be exactly transposed to the bandwagon game for all configurations of parameters. In particular, the threshold determining whether standard $Y$ 's initial performance should be considered as low or high is now given by the maximum of $c /(a+c)$ and $a / c$. This maximum is $c /(a+c)$ provided that $c /(a+c)>(\sqrt{5}-1) / 2 \approx 0.62$; in other words, it is only when the coordination benefits $(c)$ represent at least $62 \%$ of total benefits $(a+c)$ that the same taxonomy of $Y$ 's performance can be used for the negotiation and bandwagon games. In order to ease comparisons and further resolution of the game, I will by now assume that this inequality holds.
} 
- (Commit to $X$, Wait) in case $L L$, for all $\delta \in[0,1]$, and in cases $L H$ and $H H$, for $0 \leq \delta \leq \hat{\delta}_{b}$;

- (Wait, Wait) in cases LH and HH, for $\hat{\delta}_{b} \leq \delta \leq 1$.

Three instructive conclusions can be drawn from Lemma 5. (i) As in the negotiation game, very impatient players immediately coordinate on standard $X$ in every case. (ii) However, even patient players do so when $Y$ 's performance is low at the initial and at the final periods (case LL). (iii) It is only when $Y$ 's final performance is high that patient players agree to wait.

The cutoff between 'patience' and 'impatience' is given by $\hat{\delta}_{b} \equiv \sqrt{(a+c) /\left(y_{3} c\right)}$. This threshold expresses the indifference condition for player 1 between coordinating on $X$ in period 1 (yielding a payoff of $a+c$ ) or on $Y$ in period 3 (yielding a payoff of $\left.\delta^{2} y_{3} c\right)$.

\subsection{Summary}

Comparing the results of the two previous lemmas, and noting that in cases LH and $\mathrm{HH} \hat{\delta}_{b}>\bar{\delta}_{b}$, one easily finds out on which standard, and when, players coordinate in the bandwagon game. The following proposition summarizes the results.

Proposition 6 The standard adopted by the players and the timing of adoption at the RDSP equilibrium of the bandwagon game depend on the values of the parameters in the following way.

- In case LL, players adopt standard $X$ in the first period whatever their degree of patience.

- In cases LH and HH, impatient players adopt standard $X$ in the first period (i.e., for $0 \leq \delta \leq \hat{\delta}_{b}$ ) and patient players adopt standard $Y$ in the third period (i.e., for $\hat{\delta}_{b} \leq \delta \leq 1$ ).

The major difference with the negotiation game has already been emphasized: when unilateral adoption is substituted for verbal negotiation as the coordinating principle, players are more eager to coordinate as early as in period 1 (it is only in the case where they are very patient and that $Y$ 's final performance is high that 
they delay their decision up to period 3). As a result, the chances for standard $Y$ to be adopted become lower: since standard $X$ performs better at the beginning of the game, it is most often adopted by both players.

\section{$5 \quad$ Policy implications}

The objective of this section is to gauge the performance of the RDSP equilibrium of the two games. The more reasonable way to do so, in this simple model, is to compare the equilibria with the solution that would maximize the sum of the players' payoffs (in present value). It is readily checked that this benchmark involves common adoption of $X$ in period 1 if $\delta^{2} y_{3}<1$, and of $Y$ in period 3 otherwise. $^{21}$

Tables 6 to 8 summarize the results of the comparison for cases LL, LH and $\mathrm{HH}$. As before, the range of the discounting factor (i.e., the unit interval) is divided into separate areas, and for each area, the outcomes of the two games are reported. The tables read as follows: the outcomes appearing in bold face coincide with the benchmark; the outcomes appearing in italic differ from the benchmark.

Table 6: Comparison of the coordination mechanisms in case LL

\begin{tabular}{c|c|c|c|c|}
$\delta \in$ & $\begin{array}{c}\text { Area I } \\
{\left[0, \frac{c}{a+c}\right]}\end{array}$ & $\begin{array}{c}\text { Area II } \\
{\left[\frac{c}{a+c}, \frac{1}{y_{3}}\right]}\end{array}$ & $\begin{array}{c}\text { Area III } \\
{\left[\frac{1}{y_{3}}, \sqrt{\frac{1}{y_{3}}}\right]}\end{array}$ & $\begin{array}{c}\text { Area IV } \\
{\left[\sqrt{\frac{1}{y_{3}}}, 1\right]}\end{array}$ \\
\hline Negotiation & $\mathbf{X}$ in 1 & $Y$ in 2 & $Y$ in 3 & $\mathbf{Y}$ in $\mathbf{3}$ \\
\hline Bandwagon & $\mathbf{X}$ in 1 & $\mathbf{X}$ in $\mathbf{1}$ & $\mathbf{X}$ in $\mathbf{1}$ & $X$ in 1 \\
\hline
\end{tabular}

As shown by the three tables, there are various areas of discrepancy between the benchmark and the RDSP equilibrium of the two games: unless players are very impatient, each coordination mechanism might lead to an outcome that does not maximize the sum of players' discounted payoffs. In particular, it turns out

\footnotetext{
${ }^{21}$ The two incompatibility outcomes are clearly dominated. Moreover, since $\delta \leq 1, y_{1}<$ $1, y_{2}=1$, adoption of $X$ in period 1 (yielding a joint payoff of $a+2 c$ ) dominates adoption of $X$ in periods 2 or 3 , and of $Y$ in periods 1 or 2 . So, the remaining contender is adoption of $Y$ in period 3 , which yields a joint discounted payoff of $\delta^{2} y_{3}(a+2 c)$.
} 
Table 7: Comparison of the coordination mechanisms in case LH

\begin{tabular}{c|c|c|c|c|}
$\delta \in$ & {$\left[0, \sqrt{\frac{c}{y_{3}(a+c)}}\right]$} & {$\left[\sqrt{\frac{c}{y_{3}(a+c)}}, \sqrt{\frac{1}{y_{3}}}\right]$} & $\begin{array}{c}\text { Area III } \\
{\left[\sqrt{\frac{1}{y_{3}}}, \sqrt{\frac{a+c}{y_{3} c}}\right]}\end{array}$ & $\begin{array}{c}\text { Area IV } \\
{\left[\sqrt{\frac{a+c}{y_{3}}}, 1\right]}\end{array}$ \\
\hline Negotiation & $\mathbf{X}$ in $\mathbf{1}$ & $Y$ in 3 & $\mathbf{Y}$ in $\mathbf{3}$ & $\mathbf{Y}$ in $\mathbf{3}$ \\
\hline Bandwagon & $\mathbf{X}$ in $\mathbf{1}$ & $\mathbf{X}$ in $\mathbf{1}$ & $X$ in 1 & $\mathbf{Y}$ in $\mathbf{3}$ \\
\hline
\end{tabular}

Table 8: Comparison of the coordination mechanisms in case $\mathrm{HH}$

\begin{tabular}{c|c|c|c|c|c|}
$\delta \in$ & $\begin{array}{c}\text { Area I } \\
{\left[0, \hat{\delta}_{n}\right]}\end{array}$ & $\begin{array}{c}\text { Area II } \\
{\left[\hat{\delta}_{n}, \sqrt{\frac{y_{1}}{y_{3}}}\right]}\end{array}$ & $\begin{array}{c}\text { Area III } \\
{\left[\sqrt{\frac{y_{1}}{y_{3}}}, \sqrt{\frac{1}{y_{3}}}\right]}\end{array}$ & $\begin{array}{c}\text { Area IV } \\
{\left[\sqrt{\frac{1}{y_{3}}}, \sqrt{\frac{a+c}{y_{3} c}}\right]}\end{array}$ & $\left.\begin{array}{c}\text { Area V } \\
{\left[\sqrt{\frac{a+c}{y_{3} c}}\right.}\end{array}\right]$ \\
\hline Negotiation & $\mathbf{X}$ in $\mathbf{1}$ & $Y$ in 1 & $Y$ in 3 & $\mathbf{Y}$ in $\mathbf{3}$ & $\mathbf{Y}$ in $\mathbf{3}$ \\
\hline Bandwagon & $\mathbf{X}$ in $\mathbf{1}$ & $\mathbf{X}$ in $\mathbf{1}$ & $\mathbf{X}$ in $\mathbf{1}$ & $X$ in 1 & $\mathbf{Y}$ in $\mathbf{3}$ \\
\hline
\end{tabular}

that the negotiation game leads to an excessive (late) adoption of the formal standard, while the bandwagon game leads to an excessive (immediate) adoption of the de facto standard. In other words, verbal negotiation might entail too much waiting while unilateral adoption might entail excessive precipitation. Therefore, it does not make much sense to contend that one coordination mechanism performs better than the other. Nonetheless, the two mechanisms never go wrong at the same time: for every combination of parameters, the benchmark can be achieved through one or the other mechanism.

Next question which arises naturally is how to alleviate the discrepancy (supposing that side-payments between players are ruled out). In the simple model used here, only the manipulation of standard $Y$ 's performance seems to be within the grasp of a formal standards-writing organization: if more resources are devoted to the development of the formal standard, its performance can indeed be increased. Let us thus examine how this instrument should be used in the three cases.

Case LL. As shown in Table 6, the negotiation game induces two discrepancies: in Area II, players adopt standard $Y$ in period 2, in Area III, they adopt standard $Y$ in period 3, while in both instances they would jointly be better off if they 
adopted standard $X$ immediately. On the other hand, the bandwagon game gives an inappropriate outcome only when players are very patient: in Area IV, players adopt $X$ in period 1 while they would jointly be better off if they waited for adopting $Y$ in period 3 .

It turns out that the various discrepancies cannot be cured all together by a single variation of the formal standard's final performance: an increase in $y_{3}$ (with $y_{3} \leq(a+c) / c$ to remain in case LL) narrows area II but enlarges areas III and IV; conversely, a decrease in $y_{3}$ (with $y_{3}>1$ to remain in case LL) enlarges area II but narrows areas III and IV.

Case LH. Here (see Table 7), discrepancies are only observed for intermediary values of $\delta$ : in the negotiation game, players adopt standard $Y$ in period 3 (instead of standard $X$ in period 1) in Area II; in the bandwagon game, players adopt standard $X$ in period 1 (instead of standard $Y$ in period 3) in Area III.

In contrast with the previous case, it is easy to show that an increase in $y_{3}$ unambiguously narrows both discrepancy areas.

Case HH. In this case (see Table 8), there are two areas where the negotiation game does not lead to the efficient immediate adoption of standard $X$ : instead, standard $Y$ is adopted in period 1 (Area II) or in period 3 (Area III). The bandwagon game also gives rise to one discrepancy area (Area IV) where standard $X$ is adopted immediately while a late adoption of standard $Y$ would be better.

In contrast with the previous cases, two instruments are now available to alleviate the discrepancies since not only $y_{3}$, but also $y_{1}$, determine the cutoffs between the different areas. As far as final performance is concerned, it is readily checked that an increase in $y_{3}$ unambiguously narrows the three discrepancy areas. Regarding initial performance, results are less clear cut: to narrow Area II, one needs to decrease $y_{1}$, whereas to narrow Area III, one needs to increase $y_{1}$; globally, however, a decrease in $y_{1}$ narrows the range of discounting factor for which the negotiation game yields an inefficient outcome (i.e., Area II plus Area III, since $\gamma$ is a decreasing function of $y_{1}$ ).

Two other modifications of the parameters of the model could help alleviate the discrepancy between the equilibria and the benchmark:

- it can be shown that an increase in the relative value the players attach to successful coordination (i.e., an increase in $c$ with respect to a) narrows (or, 
at worse, leaves unchanged) the discrepancy areas in all three cases;

- if we are concerned with fostering the efficient adoption of the formal standard, it is obvious that any increase in the players' patience (i.e., in the discounting factor $\delta$ ) will be favored.

Of course, the relative value attached to successful coordination and the degree of patience are a matter of players' preferences. The simple model used here only indicates the direction for intervention, but suggests no means by which a standards-writing organization could achieve such intervention. A richer model should then be elaborated to endogenize these two factors and make them sensitive to policy interventions. Here follow a couple of suggestions, which are left for further research.

- One could imagine, for instance, that the relative value attached to successful coordination on a particular standard follows the evolution of the global "market share" of the standard in question; this would force us to consider that other sets of players choose between the two standards and that network externalities are at work. ${ }^{22}$

- One could also allow a standards body to shorten the time that elapses between the release of two successive versions of a formal standard (instead of considering fixed and equally spaced periods, as done here). Such efforts are indeed increasingly made in the international standards community. For instance, JTC1 ${ }^{23}$ has publicly announced in 1994 that as the focal point for IT standardization, it will provide the capability of accepting standard solutions to IT problems that have been developed outside JTC1; this has resulted in a fast-track process for the definition of $P u b$ licly Available Specifications (PAS), i.e., specifications that satisfy certain criteria, making them suitable for processing as an international standard. European standards organizations have also developed a number of similar fast track procedures.

\footnotetext{
${ }^{22}$ David and Foray (1994) develop a model that follows this line for the case of EDI standards diffusion.

${ }^{23}$ JTC 1 is the Joint Technical Committee 1 of the International Organization for Standardization (ISO) and the International Electrotechnical Commission (IEC). JTC 1's scope is standardization in the field of Information Technology.
} 


\section{Conclusion}

In the information and communications technologies (ICT) markets, standards represent much more than a technical question: they ensure the compatibility and interoperability between products of different manufacturers, and consequently the way in which all users will benefit from them. Standards may arise either out of deliberations of voluntary or governmental standards-writing organizations, or out of an unfettered market process; the former are called "formal" standards and the latter "de facto" standards.

It is widely recognized that formal standards present the following trade-off with respect to de facto standards: formal standards have a particular legitimacy and avoid the costs of adopting privately profitable, but socially undesirable, technologies; yet, in terms of the ICT industry, the timescales of their development do not always sit comfortably with the need for competitive use of the latest technology as early as possible. In a nutshell, formal standards "develop slowly but are eventually better", while de facto standards "are available sooner but might be inferior".

In such a context, it is important to understand how users choose, in a dynamic setting, between formal and de facto standards that compete with each other. To address this question, this paper has analyzed a model where two players have to choose between one formal and one de facto standard, and where both players prefer to adopt a common standard but disagree about which common standard should be adopted. Moreover, it is assumed that the de facto standard is 'mature', in the sense that it offers constant performance over the game period, while the formal standard undergoes a development process: successive versions of increasing value are released and, in particular, the formal standard performs worse, at the beginning of the game, than the de facto standard, but is known (with certainty) to perform better at the end. It is further assumed that players can either explicitly communicate and negotiate before irrevocable choices are made, or make coordination depend instead on unilateral irrevocable choices.

It turns out from the resolution of this dynamic game that the outcome crucially relies on three key factors: (i) the coordination mechanism used by the players, (ii) the relative value for the players of successful coordination, and (iii) the performance of the formal standard at the end of the game. Regarding the adoption of the formal standard, the higher the latter two values, the lower the minimal degree of patience players should have to both adopt this standard. 
In terms of comparison with the outcome that maximizes the sum of the players' discounted payoffs, the two coordination mechanisms are imperfect but entail contrasting failures: verbal negotiation leads to an excessive adoption of the formal standard, while unilateral adoption excessively favors the de facto standard. However, an increase in the final performance of the formal standard tends to alleviate these discrepancies.

Besides the extensions already outlined in the previous section, the model could be further developed along four lines in order to explore more closely the trade-off between formal and de facto standardization. A first line would consider that players are asymmetric, with respect either to their degree of patience or to their valuation of successful coordination. The second line of research would be to study the possibility of re-assessing the choice of standard (at the price of a switching cost). The third line would be to introduce uncertainty about the future performance of the formal standard. Finally, the assumption of once-andfor-all payoffs could be questioned and intermediary payoffs (which accrue to the players during the coordination process) could be introduced.

\section{Appendix. Risk-dominance relation between equilibria}

Consider the following general two-player game in strategic form in which each player has two pure strategies (see Table 9). Suppose that there are two purestrategy Nash equilibrium, say $(A, A)$ and $(B, B)$. This is the case if (i) $a_{11} \geq a_{21}$ and $b_{11} \geq b_{12}$, and (ii) $a_{22} \geq a_{12}$ and $b_{22} \geq b_{21}$.

Table 9: A general two-player game in strategic form

\begin{tabular}{c|c|c|}
\multicolumn{1}{c}{} & \multicolumn{1}{c}{$\mathbf{A}$} & \multicolumn{1}{c}{$\mathbf{B}$} \\
\cline { 2 - 3 } $\mathbf{A}$ & $a_{11}, b_{11}$ & $a_{12}, b_{12}$ \\
\cline { 2 - 3 } $\mathbf{B}$ & $a_{21}, b_{21}$ & $a_{22}, b_{22}$ \\
\cline { 2 - 3 } & &
\end{tabular}

Define the basin of attraction of a PSNE as the range of randomized strategies for which the pure strategies composing this PSNE are optimal. More formally, let $\lambda_{i}$ be the probability that player $i$ places on strategy $B$. Player 1 is indifferent 
between choosing strategy $A$ or strategy $B$ if $\lambda_{2}$ is such that:

$$
\begin{aligned}
\left(1-\lambda_{2}\right) a_{11}+\lambda_{2} a_{12} & =\left(1-\lambda_{2}\right) a_{21}+\lambda_{2} a_{22} \\
\Longleftrightarrow & \lambda_{2}=\frac{a_{11}-a_{21}}{a_{11}+a_{22}-a_{12}-a_{21}} \equiv \hat{\lambda}_{2} .
\end{aligned}
$$

Accordingly, player 1 prefers strategy $A$ [resp. $B]$ if $\lambda_{2}<$ [resp. $\left.>\right] \hat{\lambda}_{2}$.

Similarly, player 2 is indifferent between strategies $A$ and $B$ if $\lambda_{1}$ is such that

$$
\lambda_{1}=\frac{b_{11}-b_{12}}{b_{11}+b_{22}-b_{12}-b_{21}} \equiv \hat{\lambda}_{1}
$$

player 2 prefers strategy $A$ [resp. $B]$ if $\lambda_{1}<$ [resp. $\left.>\right] \hat{\lambda}_{1}$. Note that $\left(\hat{\lambda}_{1}, \hat{\lambda}_{2}\right)$ is nothing but the mixed-strategy equilibrium of the game.

Applying the definition, we have that the basin of attraction of $(A, A)$ is defined as the surface, in the unit square $\left(\lambda_{1}, \lambda_{2}\right)$, where both players prefer strategy $A$; since player $i$ prefers $A$ for values of $\lambda_{i}$ which are no greater than $\hat{\lambda}_{i}$, we have that the basin of attraction of $(A, A)$ is equal to $\hat{\lambda}_{1} \hat{\lambda}_{2}$. By analogy, the basin of attraction of $(B, B)$ is equal to $\left(1-\hat{\lambda}_{1}\right)\left(1-\hat{\lambda}_{2}\right)$. We can now define the relation of risk-dominance in terms of these basins of attraction; we will say that one equilibrium risk-dominates the other if its basin of attraction is wider that the basin of the other. In the present case, $(A, A)$ is risk-dominant iff $\$ \hat{\lambda}_{1} \hat{\lambda}_{2}>\left(1-\hat{\lambda}_{1}\right)\left(1-\hat{\lambda}_{2}\right) \Longleftrightarrow \hat{\lambda}_{1}>1-\hat{\lambda}_{2}, \$$ which is equivalent to

$$
\left(a_{11}-a_{21}\right)\left(b_{11}-b_{12}\right)>\left(a_{22}-a_{12}\right)\left(b_{22}-b_{21}\right) .
$$

Each side of this inequality represents the product of the two players' incentives for complying to a particular equilibrium when they expect that the other player is doing so as well; this is another interpretation for the basin of attraction of a particular PSNE. Naturally, if expression (1) holds with the opposite inequality, then $(B, B)$ is risk-dominant, and if it holds with equality, then there is no relation of risk dominance between the two equilibria.

\section{References}

[1] Belleflamme, P. (1999) "Assessing the diffusion of EDI standards across business communities". EURAS Yearbook of Standardization, Vol. 2, ed. by Manfred J. Holler and Esko Niskanen (Homo oeconomicus XV(3)), Munich: Accedo, 301-324. 
[2] Besen, S. and J. Farrell (1994) "Choosing how to compete: Strategies and tactics in standardization". Journal of Economic Perspectives, 8: 117-131.

[3] Cabrales, A., W. Garcia-Fontes, and M. Motta (2000) "Risk dominance selects the leader: An experimental analysis". International Journal of Industrial Organization, 18: 137-162.

[4] Cooper, R., D. DeJong, R. Forsythe and T. Ross (1989) "Communication in the battle of the sexes games: Some experimental results". RAND Journal of Economics, 20:557-568.

[5] Cooper, R., D. DeJong, R. Forsythe and T. Ross (1992) "Communication in coordination games". Quarterly Journal of Economics, 107: 739-771.

[6] Cooper, R., D. DeJong, R. Forsythe and T. Ross (1993) "Forward induction in the battle of the sexes game". American Economic Review, 83:1303-1316.

[7] David, P.A. and D. Foray (1994) "Percolation structures, markov random fields and the economics of EDI standards diffusion". In Pogorel, editor, Global telecommunication strategies and technical change (Chapter 7). Elsevier, Amsterdam.

[8] David, P.A. and S. Greenstein (1990) "The economics of compatibility standards: An introduction to recent research". Economics of Innovation and New Technology, 1: 3-41.

[9] David, P.A. and M. Shurmer (1996) "Formal standard-setting for global telecommunications and information services". Telecommunications Policy, 20: $789-815$.

[10] Economides, N. (1996) "The economics of networks". International Journal of Industrial Organization, 14:673-700.

[11] European Commission (1996) Standardization and the Global Information Society: The European Approach, Communication from the Commission to the Council and the Parliament, COM (96) 359, Brussels, 24 July (http://www.ispo.cec.be/infosoc/legreg/doc/96359.html).

[12] Farrell, J. and G. Saloner (1985) "Standardization, compatibility and innovation". Rand Journal of Economics, 16:70-83. 
[13] Farrell, J. and G. Saloner (1988) "Coordination through committees and markets". Rand Journal of Economics, 19:235-252.

[14] Foray, D. (1994) "Users, standards and the economics of coalitions and committees". Information Economics and Policy, 6:269-293.

[15] Goerke, L. and M. Holler (1995) "Voting on Standardization". Public Choice, 83:337-351.

[16] Greenstein, S. (1992) "Invisible hands and visible advisors: An economic interpretation of standardization". Journal of the American Society for Information Science, 43:538-549.

[17] Harsanyi, J. (1995) "A new theory of equilibrium selection for games with complete information". Games and Economic Behavior, 8:91-122.

[18] Harsanyi, J. and R. Selten (1988) A general theory of equilibrium selection in games. MIT Press, Cambridge (MA).

[19] Katz, M. and C. Shapiro (1994) "Systems competition and network effects". Journal of Economic Perspectives, 8:93-115.

[20] Lehr, W. (1992) "Standardization: Understanding the process". Journal of the American Society for Information Science, 43:550-555.

[21] Matutes, C. and P. Régibeau (1996) "A selective review of the economics of standardization. Entry deterrence, technological progress and international competition". European Journal of Political Economy, 12:183-209.

[22] Swann, P. and M. Shurmer (1994) "The emergence of standards in PC software: who would benefit from institutional intervention?". Information Economics and Policy, 6:295-318.

[23] Weiss, M. and C. Cargill (1992) "Consortia in the standards development process". Journal of the American Society for Information Science, 43:559565. 

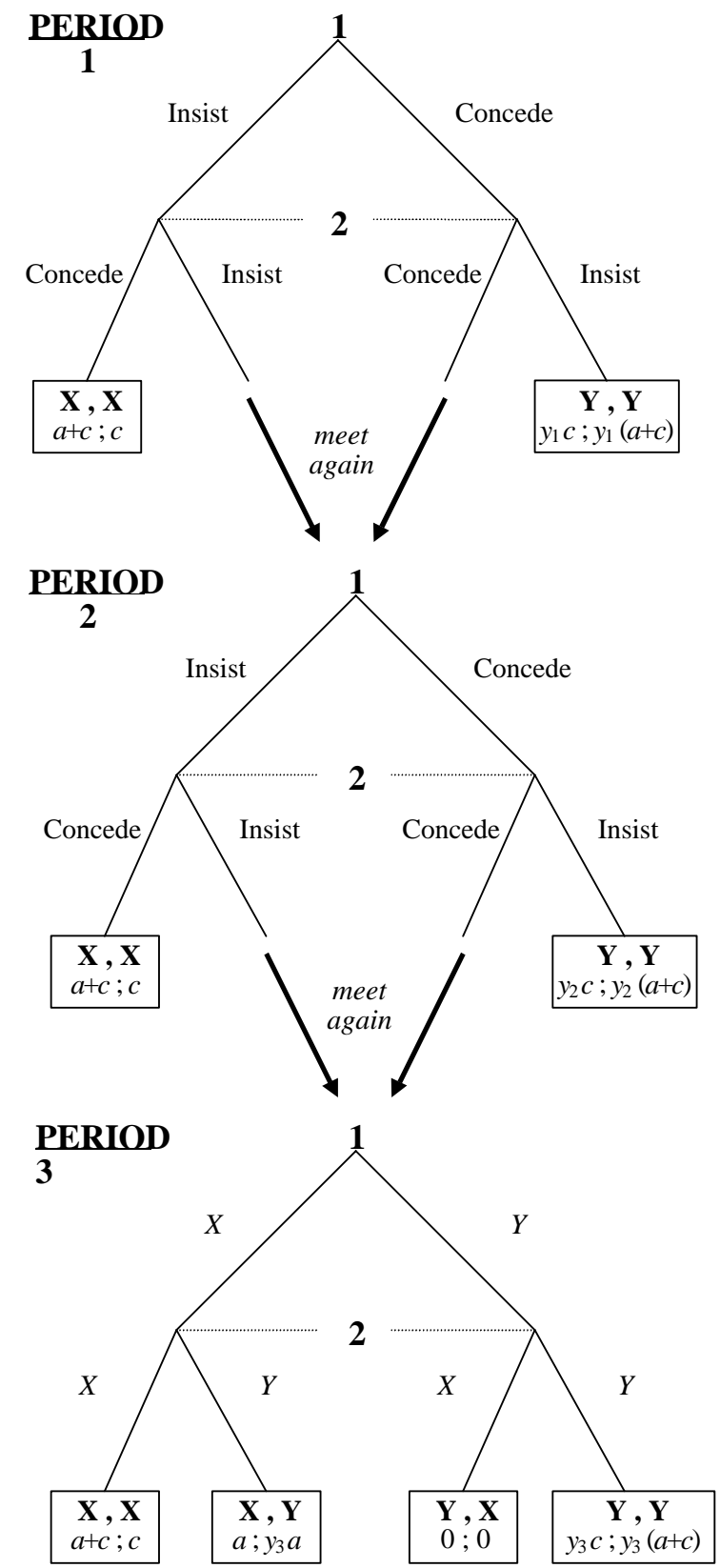

Figure 1: Game tree for the negotiation game 


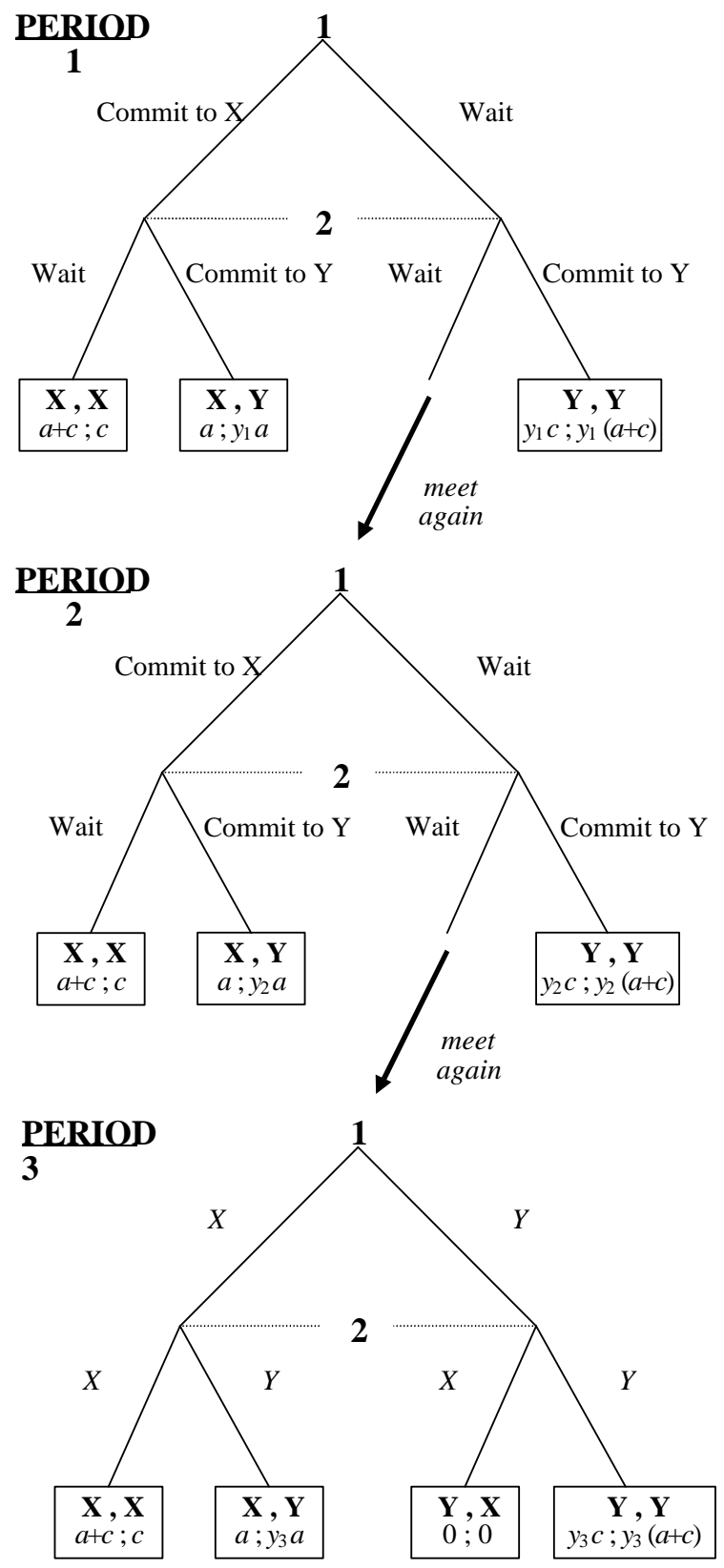

Figure 2: Game tree for the bandwagon game 